\title{
Schmerzende Gelenke
}

Degenerative Gelenkbeschwerden sind eine Crux, unter der viele Patienten zu leiden haben. Was kann man Betroffenen aus der naturheilkundlichen Therapie anbieten, wollte ein Leser wissen. Die Antwort unseres Experten macht deutlich, dass auch gegen diese Symptome durchaus das eine oder andere Kraut gewachsen ist. Sollten auch Sie Fragen zum Thema haben, schreiben Sie uns: brigitte.moreano@springer.com.

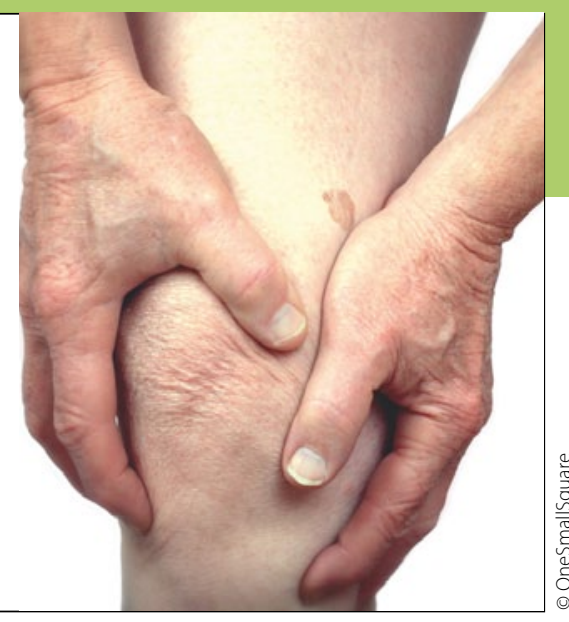

Frage:

Welche naturheilkundlichen Maßnahmen können Patienten mit degenerativen Kniebeschwerden helfen?

\section{Antwort:}

Phytotherapeutisch werden zur Schmerzlinderung und Entzündungshemmung Weidenrinden-, Teufelskrallen, Cayennepfefferextrakte oder das Kombinationspräparat aus Pappelrinde/-blättern, echtem Goldrutenkraut und Eschenrinde eingesetzt. Wegen ihrer guten Verträglichkeit und geringen Nebenwirkungsraten bieten sich Phytopharmaka als Alternative bzw. adjuvant zu chemisch-definierten Substanzen v. a. beim entzündungsassoziierten Nozizeptorenschmerz (Weidenrinden-, Teufelskrallenwurzelextrakte), beim tonusassoziierten Nozizeptorenschmerz (Capsicum-Extrakte topisch) und bei psychosomatisch-psychosozialer Ursache (Johanniskrautextrakt) an. Phytopharmaka sind bzgl. ihres Wirkprofils und ihrer klinischen Wirksamkeit durch prospektive, randomisierte, kontrollierte Doppelblindstudien gegenüber Placebo und chemischdefinierten Referenzsubstanzen untersucht, sodass sie evidenzbasiert und sicher eingesetzt werden können.

Die innere Anwendung kann mit Beinwellpackungen (abends) oder Beinwellsalbe (tagsüber) kombiniert werden. Ergebnisse neuerer Forschung zeigen, dass Moorinhaltsstoffe - wie viele Schmerzmedikamente - agonistisch auf alpha-2-Rezeptoren wirken. Besonders bei Kniegelenksarthosen kommen daher auch Moorbäder und Moorextrakte in Betracht.

Als sehr wirkungsvoll zeigt sich unserer klinischen Erfahrung nach die Blutegelbehandlung, die heute auch evidenzbasiert einsetzbar ist. Nach entsprechender Schulung kann sie auch in der niedergelassenen Praxis durchgeführt werden. In der Abteilung Naturheilkunde der Klinik Blankenstein verabreichen wir im Rahmen der naturheilkundlichen stationären Komplexbehandlung ca. 3000 Blutegel pro Jahr. Viele Patienten, für die die Orthopäden als einzige verbleibende Therapieoption den alloplastischen Gelenkersatz sahen, wurden hierunter ohne Operation für viele Monate nahezu beschwerdefrei.

Von hoher Bedeutung sind ordnungstherapeutische Hinweise auf ausreichende Bewegung ohne Überlastung (Schwimmen oder Fahrradfahren).

Besonders bei übergewichtigen Patienten kann im Zusammenspiel von Ernährungstherapie, Bewegungstherapie (,je mehr Bewegung, desto mehr Kalorien kann man sich leisten") und Ordnungstherapie (z. B. maßvoller Umgang mit Genussmitteln) auf eine Gewichtsreduktion und dadurch Entlastung der Gelenke der unteren Extremitäten und der Wirbelsäule hingewirkt werden.
Frage:

Welche Therapien bieten sich bei der schmerzhaften Schulter an?

\section{Antwort:}

Die naturheilkundliche Therapie richtet sich nach der jeweiligen Ursache. Rotatorenmanschettenrupturen, Kalkschulter, Schultereckgelenkarthrosen, Insertionstendopathien müssen unterschiedlich behandelt werden. Hier sind auch die Grenzen der Naturheilverfahren zu beachten.

Bei den meisten chronischen Schultererkrankungen steht die Krankengymnastik im Vordergrund. Wichtig ist, den Betroffenen Übungen zu erklären, die sie täglich in Eigenverantwortung durchführen können. Bei Insertionstendopathien kann Elektrotherapie in Form einer gepulsten Ultraschalltherapie dreimal pro Woche zum Einsatz kommen. Schröpftherapien, Blutegeltherapien und Akupunktur bieten sich ergänzend an. Eine wichtige Rolle spielt die Neuraltherapie. Bei der kleinen Neuraltherapie werden Quaddelungen im Bereich des Schultergelenks durchgeführt. Bei der „großen Neuraltherapie" werden Injektionen an den M. subscapularis, an den N. suprascapularis und an das Sternoklavikulargelenk mit einem Neuraltherapeutikum (z.B. Procain) verabreicht.

Eine begleitende Phytotherapie mit Weidenrinden- oder Teufelskrallenextrakt ist hilfreich.

Prof. Dr. A.-M. Beer, Hattingen = 